\title{
Why You Should Charge Your Friends for Borrowing Your Stuff
}

\author{
Kijung Shin, Euiwoong Lee, Dhivya Eswaran, and Ariel D. Procaccia \\ Carnegie Mellon University, Pittsburgh, PA, USA \\ \{kijungs, euiwoonl, deswaran, arielpro\}@ cs.cmu.edu
}

\begin{abstract}
We consider goods that can be shared with $k$-hop neighbors (i.e., the set of nodes within $k$ hops from an owner) on a social network. We examine incentives to buy such a good by devising game-theoretic models where each node decides whether to buy the good or free ride. First, we find that social inefficiency, specifically excessive purchase of the good, occurs in Nash equilibria. Second, the social inefficiency decreases as $k$ increases and thus a good can be shared with more nodes. Third, and most importantly, the social inefficiency can also be significantly reduced by charging free riders an access cost and paying it to owners, leading to the conclusion that organizations and system designers should impose such a cost. These findings are supported by our theoretical analysis in terms of the price of anarchy and the price of stability; and by simulations based on synthetic and real social networks.
\end{abstract}

\section{Introduction}

Social networks are known to play an important role in the everyday choices people make. In particular, a significant body of work studies the network effect, in which there are payoffs from aligning one's decision with those of others [Markus, 1987; Blume, 1993; Ellison, 1993; Rogers, 2010]. For example, direct payoffs arise when friends or collaborators use compatible technologies instead of incompatible ones, that is, the game rewards coordination.

Our work considers the purchase of shareable goods, which, in a sense, gives rise to a certain type of anticoordination game. Indeed, buying such a good yields a benefit only when no friend buys the good, since otherwise free riding is possible. An example that would be familiar to most parents is seldom-used baby gear, such as portable cribs, which are frequently borrowed by friends or friends of friends; similar examples include ski gear and hiking equipment. Expensive lab equipment provides a more pertinent example: Confocal laser scanning microscopes, or polymerase chain reaction (PCR) machines, are typically bought by one investigator and used by collaborators. In the realm of AI, one can imagine a multi-agent system populated by heterogeneous software agents that interact and share special compu- tational resources, e.g., a high-end graphics processing unit (GPU) for particularly demanding image processing tasks.

To examine incentives to buy shareable goods, we devise game-theoretic models where each node on a network decides whether to buy a good that is shareable with $k$-hop neighbors (i.e., nodes within $k$ hops from an owner), or free ride. Specifically, the good in question is non-excludable and nonrivalrous in that no $k$-hop neighbor can be excluded from use, and use by a neighbor does not reduce availability to others. Note that the goods in the examples given above are (essentially) non-rivalrous, as any single person (or agent) requires the good only from time to time.

We find that social inefficiency, specifically excessive purchase of the good, occurs in Nash equilibria. Moreover, the social inefficiency decreases as $k$ increases and thus a good is shared with more people. Finally, charging free riders an access cost and paying it to owners also significantly reduces the social inefficiency. We support these findings both theoretically and experimentally.

Compared with previous work on shareable goods on a network, discussed in Section 5, our contributions are as follows:

- Efficiency Analysis of Equilibria : We provide worstcase analysis of the efficiency of equilibria, in terms of the price of anarchy and the price of stability.

- Simulation on Real-world Networks: The simplicity of our model allows us to measure social inefficiency on real-world social networks, with thousands of nodes, through simulations of best-response dynamics.

- Mechanism Design: We analyze the effects of access costs on social inefficiency and suggest an appropriate cost for minimizing social inefficiency.

The rest of the paper is organized as follows. In Section 2, we define shareable goods games on a network. In Section 3, we give a theoretical analysis of the efficiency of equilibria. In Section 4, we present simulation results. After discussing related work in Section 5, we draw conclusions in Section 6.

\section{Our Models}

In this section, we formally define two game-theoretic models of the purchase of shareable goods on a network.

\subsection{Shareable Goods Game (SGG)}

Consider an undirected network $\mathcal{G}=(\mathcal{V}, \mathcal{E})$ where $\mathcal{V}=$ $\{1,2, \ldots, n\}$. The players of the game are nodes in $\mathcal{G}$. Each 
Table 1: Utility in an SGG.

\begin{tabular}{|c|c|c|c|}
\hline state & \multicolumn{2}{|l|}{ conditions } & utility (i.e., $u_{i}$ ) \\
\hline $\begin{array}{c}\text { buy } \\
\text { free ride } \\
\text { no access }\end{array}$ & \multicolumn{2}{|c|}{$\begin{array}{c}s_{i}=1 \\
s_{i}=0, \sum_{j \in \mathcal{N}_{i}(k)} s_{j} \geq 1 \\
s_{i}=0, \sum_{j \in \mathcal{N}_{i}(k)} s_{j}=0\end{array}$} & $\begin{array}{c}b-p(>0) \\
b(>0) \\
0\end{array}$ \\
\hline \multicolumn{4}{|c|}{ Table 2: Utility in an SGG-AC. } \\
\hline state & conditions & \multicolumn{2}{|c|}{ utility (i.e., $u_{i}$ ) } \\
\hline $\begin{array}{c}\text { buy } \\
\text { rent } \\
\text { no access }\end{array}$ & $\begin{array}{c}s_{i}=i \\
s_{i}=j(\neq i), s_{j}=j \\
s_{i}=j(\neq i), s_{j} \neq j\end{array}$ & \multicolumn{2}{|c|}{$\begin{array}{c}b-p+a\left|\mathcal{F}_{i}\right|(>0) \\
b-a(>0) \\
0\end{array}$} \\
\hline
\end{tabular}

node decides whether to buy a good or not. The strategy of node $i$ is denoted by $s_{i} \in \mathcal{S}_{i}$, where $\mathcal{S}_{i}=\{0,1\}$ denotes the strategy set of node $i$. If node $i$ buys the good then $s_{i}=1$, and otherwise $s_{i}=0$ (only pure strategies are considered). Given any strategy profile $s=\left(s_{1}, s_{2}, \ldots, s_{n}\right)$, we use $s_{-i}$ to denote the strategies taken by all nodes but $i$. Then, $s$ is also denoted by $\left(s_{i}, s_{-i}\right)$. The price of a good is $p(>0)$, which is identical for all nodes. A node gets benefit $b(>p)$ by having access to a good and 0 otherwise. Each node $i$ can access a good if it buys the good itself or has at least one node who buys the good within $k(\geq 1)$ hops. We assume that having access to multiple goods does not increase the benefit of a node and that being accessed by multiple nodes does not decrease the benefit derived from a good (non-rivalry).

In this setting, the utility $u_{i}(s)$ of node $i$ under strategy profile $s$ depends on the strategies of its $k$-hop neighbors $\mathcal{N}_{i}(k)$ (i.e., the set of nodes within $k$-hops from $i$ including $i$ itself), as given in Table 1 . Note that each node gets the highest utility $b$ when it free rides and the second highest utility $b-p$ when it buys the good. Each node gets the lowest utility 0 when neither the node nor its $k$-hop neighbors buy the good.

SGG extends the best-shot game [Hirshleifer, 1983], which is equivalent to SGG if $k=1$, by considering not only direct but $k$-hop neighbors. SGG-AC, discussed in the following subsection, further extends SGG by considering access costs.

\subsection{Shareable Goods Game with Access Costs (SGG-AC)}

In this subsection, we extend the game defined in the previous section to a game we call the shareable goods game with access costs (SGG-AC), where each free rider has to pay an access cost. We focus on the differences from an SGG.

The strategy set of each node $i$ is $\mathcal{S}_{i}=\mathcal{N}_{i}(k)$, its $k$-hop neighbors including $i$ itself. If node $i$ buys a good then $s_{i}=i$, and if node $i$ does not buy a good but wants to access a good bought by node $j \neq i$ then $s_{i}=j$. If $s_{i}=j$ for $j \neq i$, and node $j$ actually buys a good (i.e., $s_{j}=j$ ) then node $i$ derives benefit from the good at the expense of paying an access cost of $a(<p)$ to node $j$. The followers of node $i$, the set of nodes who want to access the good bought by node $i$, are denoted by $\mathcal{F}_{i}=\left\{j \in \mathcal{N}_{i}(k) \backslash\{i\}: s_{j}=i\right\}$. Then, the utility $u_{i}(s)$ of node $i$ under strategy profile $s$ in an SGG-AC is given in Table 2. Define the follower threshold $\xi=\lceil p / a\rceil-1$; for ease of exposition we assume that $p / a$ is not an integer. If node $i$
Buy $O$ Not Buy

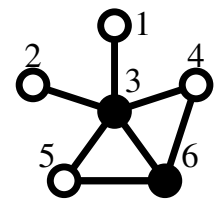

(a) Not NE

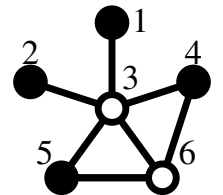

(b) $\mathrm{NE}(\operatorname{cost}=4 p)$

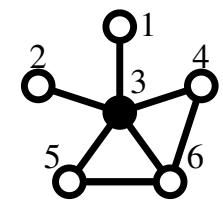

(c) $\mathrm{NE}($ cost $=p)$
Figure 1: Example strategy profiles in an SGG when $k=1$. (a) is not an NE since each of node 3 and node 6 would be better off not buying. Between the NEs, (c) leads to a lower social cost than (b).

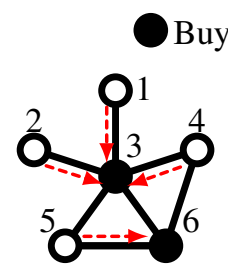

(a) Not NE

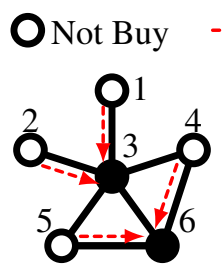

(b) $\mathrm{NE}(\operatorname{cost}=2 p)$

\section{Access}

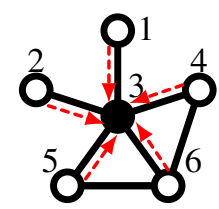

(c) $\mathrm{NE}(\cos t=p)$
Figure 2: Example strategy profiles in an SGG-AC when $k=$ 1 and $\xi=2$. Arrows indicate who accesses whose products. (a) is not an NE since node 6 is better off not buying. Between the NEs, (c) leads to a lower social cost than (b).

has at least $\xi$ followers (i.e., $\left|\mathcal{F}_{i}\right| \geq \xi$ ), it has the highest utility when it buys a good (i.e., $s_{i}=i$ ). Otherwise, renting a good is preferred. Each node has the lowest utility when it is not accessing any good.

\section{Analysis of Equilibria}

In this section, we define equilibria in the games described in Section 2. Then, we analyze the efficiency of the equilibria in terms of price of anarchy ( $\mathrm{PoA})$ and price of stability $(\mathrm{PoS})$.

\subsection{Definition and Existence of Equilibria}

We use the ubiquitous concept of Nash equilibrium (NE) as our solution concept. We first formally define it.

Definition 1 (Nash Equilibrium). A strategy profile $s=$ $\left(s_{i}, s_{-i}\right)$ is a Nash equilibrium (NE) if no node can increase its utility by changing its strategy given the strategies of the other nodes, i.e.,

$$
\forall i \in \mathcal{V}, \forall s_{i}^{\prime} \in \mathcal{S}_{i}, u_{i}\left(\left(s_{i}, s_{-i}\right)\right) \geq u_{i}\left(\left(s_{i}^{\prime}, s_{-i}\right)\right) .
$$

Figures 1 and 2 give examples of NEs in an SGG and an SGG-AC, respectively, with explanations. Note that a strategy profile is an NE in an SGG if and only if the set of owners is a $k$-independent dominating set [Kreuter and Nierhoff, 1997], i.e., any two owners have distance at least $k+1$ and every node has distance at most $k$ to some owner. Theorem 1 states that an NE always exists in both games.

Theorem 1 (Existence of Nash Equilibria). An NE exists in any $S G G$ and $S G G-A C$.

Proof. Given $\mathcal{G}=(\mathcal{V}, \mathcal{E}), k$ and $\xi$, the following procedure gives an NE $s$ for any SGG and $s^{\prime}$ for any SGG-AC. Choose an arbitrary node $i$ in the graph, let $i$ buy a good $\left(s_{i}=1\right.$, $s_{i}^{\prime}=i$ ), and for each node $j$ within $k$ hops from $i$, let $j$ follow $i\left(s_{j}=0, s_{j}^{\prime}=i\right)$. Delete $i$ and all nodes within $k$ hops from 
Proceedings of the Twenty-Sixth International Joint Conference on Artificial Intelligence (IJCAI-17)

Table 3: Summary of our analysis of efficiency of equilibria.

\begin{tabular}{c|c|c|c}
\hline & & in SGG & in SGG-AC \\
\hline \multirow{2}{*}{$k=1$} & $\begin{array}{c}\text { PoA } \\
\text { PoS }\end{array}$ & $\Theta(n)$ & $\Theta(\xi)(=\mathbf{1}$ if $\xi \leq 2)$ \\
\hline \multirow{2}{*}{$k>1$} & $\begin{array}{c}\text { PoA } \\
\text { PoS }\end{array}$ & $\Theta(n / k)$ & $\Theta(\xi / k)(=1$ if $\xi \leq 2\lfloor k / 2\rfloor+1)$ \\
\hline
\end{tabular}

$i$, and repeat until there is no node left in $\mathcal{G}$. At the end, every node either buys a good or accesses its $k$-hop neighbor's.

Each node that accesses its $k$-hop neighbor's good cannot increase its utility by buying a good since the utility of accessing its $k$-hop neighbor's is greater than that of buying (and no one follows). Each node $i$ that buys a good also cannot increase its utility by following another node because the procedure ensures that there is no node that buys a good and is within distance $k$ from $i$. Therefore, $s$ and $s^{\prime}$ are NEs for the given SGG and SGG-AC, respectively.

\subsection{Social Inefficiency in Equilibria}

We now turn to the analysis of NEs in our games. It is important to note that a node that does not access any good can increase its utility by buying a good, without decreasing the utilities of the others in both of our games (see Tables 1 and 2 ). Thus, if we let $\mathcal{T}$ be the set of strategy profiles where every node accesses a good and thus gets benefit $b^{1}$, then all NEs belong to $\mathcal{T}$. Due to the same reason, every socially optimal strategy profile (i.e., strategy profile $s$ maximizing social welfare $\left.\sum_{i \in \mathcal{V}} u_{i}(s)\right)$ belongs to $\mathcal{T}$. Therefore, to define PoA and $\mathrm{PoS}$, we only need to consider the strategy profiles in $\mathcal{T}$. Since all strategy profiles in $\mathcal{T}$ have the same sum of benefits, we can compare them simply by their social cost, which is proportional to the number of nodes buying a good (see Definition 2). Importantly, access costs in SGG-AC cancel out (they are paid by some players to others) and do not affect the social cost.

Definition 2 (Social Cost). Given a graph $\mathcal{G}=(\mathcal{V}, \mathcal{E})$, the social cost of a strategy profile $s=\left(s_{1}, \ldots, s_{n}\right) \in \mathcal{T}$ is the sum of prices paid by the nodes, i.e.,

$$
\operatorname{cost}(s)= \begin{cases}p \cdot\left|\left\{i \in \mathcal{V}: s_{i}=1\right\}\right| & \text { in } S G G \\ p \cdot\left|\left\{i \in \mathcal{V}: s_{i}=i\right\}\right| & \text { in } S G G-A C .\end{cases}
$$

The price of anarchy (PoA) is defined as the social cost of the worst NE divided by minimum social cost (see Definition 3 ) and the price of stability $(P o S)$ is defined as the social cost of the best NE divided by minimum social cost (see Definition 4). Large PoA and PoS indicate that NEs are socially inefficient.

Definition 3 (Price of Anarchy). Given a graph $\mathcal{G}=(\mathcal{V}, \mathcal{E})$ with $n$ nodes, the price of anarchy (PoA) is defined as

$$
\mathrm{PoA}=\frac{\max _{s \in \mathcal{T}}: \text { s is an } N E \operatorname{cost}(s)}{\min _{s \in \mathcal{T}} \operatorname{cost}(s)} .
$$

Definition 4 (Price of Stability). Given a graph $\mathcal{G}=(\mathcal{V}, \mathcal{E})$ with $n$ nodes, the price of stability $(\mathrm{PoS})$ is defined as

$$
\mathrm{PoS}=\frac{\min _{s \in \mathcal{T}}: \text { s is an } N E \operatorname{cost}(s)}{\min _{s \in \mathcal{T}} \operatorname{cost}(s)} .
$$

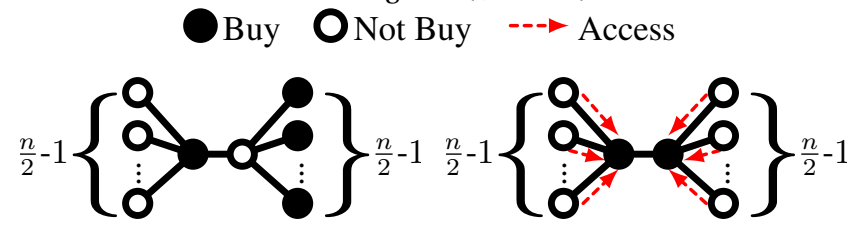

(a) Best NE in SGG

(b) Best NE in SGG-AC

Figure 3: An example of social inefficiency in an SGG. Assume $k=1$. Arrows indicate who accesses whose products. In this example graph, the best NE in an SGG is (a), whose social cost is $n p / 2$. In an SGG-AC (with $\xi \leq n / 2-1$ ), however, the best NE is (b), whose social cost is $2 p$, equal to the minimum social cost.

In Table 3, we summarize the results of our worst-case efficiency analysis. That is, we analyze the two measures in the worst case over all graphs. As stated in Theorem 2, both the PoA and PoS in SGGs are $\Theta(n / k)$ in the worst case. That is, not only worst NEs but also best NEs can be severely inefficient, with social cost as high as $n / k$ times the optimum. Figure 3(a) shows an example of such inefficiency for $k=1$, where even the best NEs in the SGG have social cost $n p / 2$, while the minimum social cost, in Figure 3(b), is $2 p$.

Theorem 2 (PoA and PoS in SGG). PoA and PoS in SGG are both $\Theta(n / k)$ in the worst case.

Proof. For the upper bound on PoA, fix an arbitrary NE $s \in$ $\mathcal{T}$. Let $k^{\prime}=\lfloor k / 2\rfloor$, and for each node $i \in \mathcal{V}$ that buys a good, consider $\mathcal{N}_{i}\left(k^{\prime}\right)$, the set of nodes within distance $k^{\prime}$ from $i$ (called a ball around $i$ ). Since each pair of nodes that buy a good are at distance at least $k+1$ from each other, these balls are pairwise disjoint.

Call a ball $\mathcal{N}\left(k^{\prime}\right)$ big if it has at least $k^{\prime}$ nodes, and small otherwise. If a ball $\mathcal{N}_{i}\left(k^{\prime}\right)$ is small, $i$ is in a connected component with less than $k^{\prime}$ nodes, and there is no other node that buys a good in that component. Let $c$ be the number of connected components in $\mathcal{G}$; there are at most $n / k^{\prime}$ big balls and $c$ small balls. The number of nodes that buy a good is equal to the number of balls, which is at most $n / k^{\prime}+c$.

Since the optimal social cost is at least $c p$, the ratio between the social cost of $s$ and the optimum is at most $\left(n / k^{\prime}+\right.$ c) $p /(c p) \leq n / k^{\prime}+1=O(n / k)$.

For the lower bound on PoS, given integers $k, m \geq 1$, consider a tree $\mathcal{G}=(\mathcal{V}, \mathcal{E})$ where there are two center nodes 1 and 2 , and $2 m$ simple paths with $k$ nodes. For $m$ of them (called left arms), one of two endpoints is connected to 1. For the other $m$ of them (called right arms), one of two endpoints is connected to 2 . Finally, 1 and 2 are connected. Figure 3 shows such a graph with $k=1$ and $m=n / 2-1$. It is easy to see that the optimal social cost is at most $2 p$, since if 1 and 2 buy a good, all nodes can access at least one good.

We claim that any NE has social cost at least $m p$. Fix an NE $s$. In $s$, either 1 or 2 does not buy a good, since they can access each other's goods. Without loss of generality, suppose that 1 does not buy a good. Consider a left arm, specifically the endpoint of the arm not connected to 1 . The only nodes within distance $k$ from this endpoint are 1 and the nodes in the same arm. Since 1 does not buy a good,

\footnotetext{
${ }^{1}$ In SGG, $s \in \mathcal{T} \Leftrightarrow \forall i \in \mathcal{V}, \sum_{j \in \mathcal{N}_{i}(k)} s_{j} \geq 1$. In SGG-AC, $s \in \mathcal{T} \Leftrightarrow \forall i \in \mathcal{V},\left(s_{i}=i\right.$ or $\left(s_{i}=j\right.$ and $\left.\left.s_{j}=j\right)\right)$.
} 
there must be a node in the same arm who buys a good. This argument holds for each left arm, so at least $m$ nodes buy a good, establishing the claim.

Since the optimal social cost is at most $2 p$ and any NE has social cost at least $m p, \operatorname{PoS} \geq m p /(2 p)=\Omega(n / k)$. Since PoS $\leq$ PoA, the theorem holds.

Intuitively speaking, the main reason for the inefficiency of NEs in SGGs is that high-degree nodes (i.e., nodes with many $k$-hop neighbors) are less likely to buy goods even when many neighbors can benefit from goods bought by highdegree nodes. Indeed, high-degree nodes are more likely to have a neighbor buying a good, and thus choose to free ride.

To incentivize high-degree nodes to buy goods, we can force their neighbors who access the good to pay an access fee to the node - as we do in SGG-ACs. In Figure 3(b), for example, the two high-degree nodes in the center still buy goods, even when they can free ride, since they receive access fees from $\xi$ or more followers, minimizing social cost.

This improvement through access costs is formalized and generalized in Theorem 3, where we show that the worst-case PoS in SGG-ACs is $\Theta(\xi / k)$, which is significantly smaller than $\Theta(n / k)$ in SGGs. In particular, if $\xi \leq \max (2\lfloor k / 2\rfloor+$ $1,2)$, then the PoS in SGG-ACs is 1, i.e., the social cost in the best equilibria is always optimal even in the worst case. Among $\xi$ values satisfying the condition, the largest one (i.e., $\xi=\max (2\lfloor k / 2\rfloor+1,2))$ is preferred to minimize the PoA, which is inversely proportional to $\xi$, as shown by Theorem 4 .

Theorem 3 (PoS in SGG-AC). PoS in SGG-ACs is $\Theta(\xi / k)$ in the worst case. In particular, it is 1 (i.e., there are guaranteed to be socially optimal equilibria) if $\xi \leq \max (2\lfloor k / 2\rfloor+1,2)$.

Proof. For the upper bound, given $\mathcal{G}=(\mathcal{V}, \mathcal{E}), k$, and $\xi$, let $\mathcal{A}^{*} \subseteq \mathcal{V}$ be a smallest set of the nodes such that for each node $i \in \mathcal{V}$, there is a node $j \in \mathcal{A}^{*}$ such that $i$ and $j$ are within distance $k$ from each other (i.e., $\left|\mathcal{A}^{*}\right| \cdot p$ is the optimal social cost). Consider a strategy profile $s$ where each node in $\mathcal{A}^{*}$ buys a good, and all other nodes access a good of a node in $\mathcal{A}^{*}$ such that for each $i \in \mathcal{A}^{*}$, the set of nodes that access $i$ 's good induces a connected subgraph. Call a node an owner if it buys a good. An owner $i$ is called a rich owner if $\left|\mathcal{F}_{i}\right| \geq \xi$, and a poor owner otherwise. That is, an owner $i$ is a rich owner if and only if $u_{i}(s)$ is at least the utility of renting a good. Note that $s \in \mathcal{T}$ is not an NE if and only if there is a poor owner who can access a good of another owner.

From $s$, we show how to construct an NE whose social cost is at most $\Theta(\xi / k) \cdot\left|\mathcal{A}^{*}\right| \cdot p$. If there is a poor owner $i$ who can follow another owner $j$, let $i$ follow $j$. For each node who previously followed $i$, if it can follow another owner, let it follow that owner. Call a node underprivileged if it previously followed $i$ but cannot follow any other owner. Scan the list of underprivileged nodes sequentially. When $\ell$ is considered, let $\ell$ be an owner (call it a new owner) and be followed by all still underprivileged nodes who can follow $\ell$ (and remove them from the underprivileged nodes list). At the end of this loop no node is left underprivileged. Repeat until no poor owner $i$ can follow another owner $j$.

One of the invariants of this procedure is that any new owner $\ell$ can never access a good of any other owner $-\ell$ became a new owner since it could not access a good of any other owner, and, subsequently, any node $i \in \mathcal{N}_{\ell}(k)$ cannot become a new owner, as it can follow $\ell$, i.e., $\ell \in \mathcal{N}_{i}(k)$. Therefore, this procedure always terminates, after having at most $\left|\mathcal{A}^{*}\right|$ owners follow other owners. The final strategy after termination is an NE since there is no underprivileged node and no poor owner who can follow another owner.

To bound the number of new owners, note that when an owner $i \in \mathcal{A}^{*}$ deviated to follow another owner $j$, among $i$ 's previous followers including $i$ (call them $\mathcal{C}_{i}$ ), at most $\max \left(1,\left\lfloor\frac{\xi}{\lfloor k / 2\rfloor+1}\right\rfloor\right)$ new owners can be created. This is because $\left|\mathcal{C}_{i}\right| \leq \xi$ (since $i$ was a poor owner) and if we let $k^{\prime}=$ $\lfloor k / 2\rfloor$, and consider the ball $\mathcal{N}_{\ell}\left(k^{\prime}\right)$ around each new owner $\ell$, $\left|\mathcal{N}_{\ell}\left(k^{\prime}\right) \cap \mathcal{C}_{i}\right| \geq \min \left(k^{\prime}+1,\left|\mathcal{C}_{i}\right|\right)$ and all balls are pairwise disjoint (all new owners are at distance at least $k+1$ from each other). The deviation of $i$ creates at most $\max \left(1,\left\lfloor\frac{\xi}{k^{\prime}+1}\right\rfloor\right)$ new owners. Therefore, the number of owners in the final NE is at most $\left|\mathcal{A}^{*}\right| \cdot \max \left(1,\left\lfloor\frac{\xi}{k^{\prime}+1}\right\rfloor\right)=O\left(\frac{\xi}{k} \cdot\left|\mathcal{A}^{*}\right|\right)$. If $\xi \leq 2 k^{\prime}+1$, $\max \left(1,\left\lfloor\frac{\xi}{k^{\prime}+1}\right\rfloor\right)=1$ so the resulting NE is a social optimum. The same conclusion holds when $\xi \leq 2$ since one deviation creates at most one new owner.

For the lower bound of $\Omega(\xi / k)$, for any integers $k, \xi$ such that $m=\frac{\xi-1}{k}$ is an integer, build the same tree as in the proof of Theorem 2. As before, the optimal social cost is $2 p$, and the social cost at NEs is at least $m p$. To see why the latter claim holds, note that (as before) 1 and 2 cannot simultaneously be owners because the total number of nodes other than 1 and 2 is $2(\xi-1)$, so that at least one of 1 and 2 must be a poor owner. Using the same argument as before, the number of owners is at least $m=\frac{\xi-1}{k}$, and $\operatorname{PoS} \geq \frac{\xi-1}{2 k}=\Omega\left(\frac{\xi}{k}\right)$.

Theorem 4 (PoA in SGG-AC). PoA in SGG-ACs is $\Theta$ (max $(n / k, n / \xi))$ in the worst case.

The proof of Theorem 4 is relegated to the full version of the paper [Shin et al., 2017]. Note that PoA in SGG-AC has the same order as PoA in SGG if $\xi=\Omega(k)$.

\section{Experiments}

In this section, we design and conduct experiments to measure social inefficiency in equilibrium, and the effects of $k$ and $\xi$ on the inefficiency. On a high level, our experiments support our qualitative theoretical conclusions.

\subsection{Algorithms}

We use best-response dynamics [Matsui, 1992], described in Algorithm 1, to find NEs. A strategy of node $i$ is a best response if it maximizes the utility of $i$ given the others' strategies. In best-response dynamics, nodes iteratively deviate to a best response, until an NE is reached. We run best-response dynamics starting from a strategy profile where no node buys a good. In both our games, best-response dynamics always converges to an NE, as formalized in Theorem 5.

Theorem 5 (Convergence of Best-Response Dynamics). For both SGGs and SGG-ACs, Algorithm 1 converges to an NE after at most three while loops in lines 5-10.

Proof. Assume first that the game is an SGG-AC. Given a strategy profile $s$, call a node underprivileged if it is not accessing a good (it may have neighbors buying a good), and 


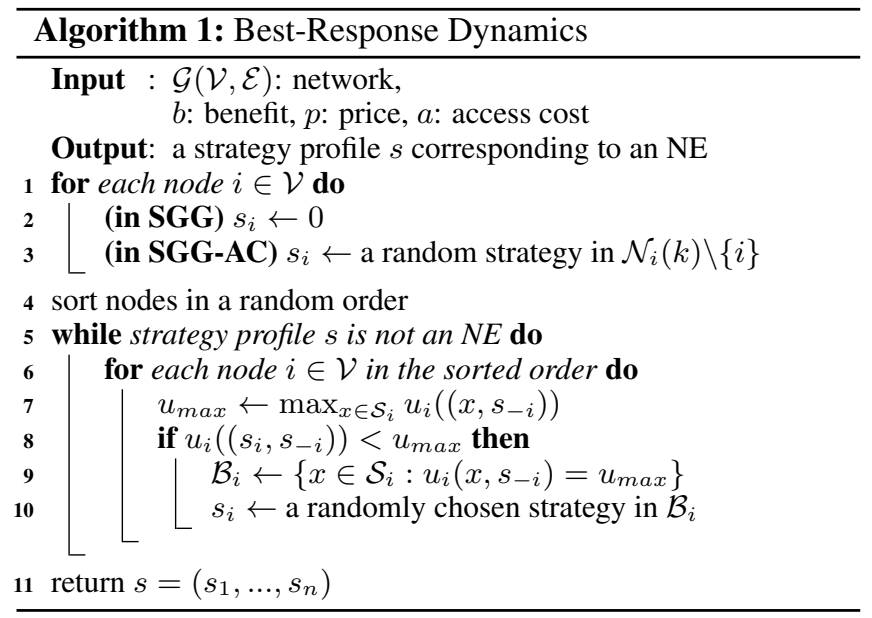

call a node an owner if it buys a good. There are four types of possible deviations a node can use to improve its utility.

Case 1. An underprivileged node buys a good since it has no $k$-hop neighbor buying a good.

Case 2. An underprivileged node rents a good (free rides in SGG) since it has a $k$-hop neighbor buying a good but has at most $\xi-1$ followers.

Case 3. A non-owner buys a good, even though it has a $k$ hop neighbor buying a good, because it has at least $\xi$ followers (who were underprivileged).

Case 4. An owner changes its action to renting (free riding in SGG) since it has a $k$-hop neighbor buying a good but has at most $\xi-1$ followers.

In SGGs, only Cases 1 and 4 are possible, ignoring the conditions on the number of followers for Case 4 . We now prove that after at most 3 iterations of the while loop, $s$ is an NE.

Claim 1. After the first iteration, Case 3 cannot happen.

Proof. Assume for contradiction that Case 3 happened for a node $i$ after the first iteration. Right before this, $i$ did not own a good, so either $i$ never owned a good, or it owned a good (via Case 1 or 3) and rented a good (free rode in SGG) later (via Case 4). Note that a node cannot get any more followers while it does not own a good. If $i$ never owned a good, it means all its followers followed it from the beginning, so $i$ should have bought a good in the first iteration, leading to contradiction. If $i$ once owned a good but decided to rent a good (free ride in SGG) later via Case 4, the number of its followers at the moment of the decision was at most $\xi-1$, and it could not have gained additional followers since then - again leading to a contradiction.

Claim 2. After the second iteration, Case 4 cannot happen.

Proof. Assume for contradiction that Case 4 happened for a node $i$ after the second iteration, which means that $i$ gave up its good and decided to rent a good (free ride in SGG). Note Case 1 does not apply to $i$ 's $k$-hop neighbors when $i$ owned the item, and by Claim 1, even Case 3 does not apply after the first iteration. This implies after the first iteration, no new owner appeared among $i$ 's $k$-hop neighbors, and the number of $i$ 's followers only increased. Thus, either Case 4 should have applied to $i$ earlier, or $i$ should not have bought the good (Case 3 did not apply), leading to a contradiction.

Claim 3. After the third iteration, Case 1 and Case 2 cannot happen.

Proof. If a node is underprivileged after the third iteration, Case 4 must have happened in the third iteration or later, contradicting Claim 2.

Combining all the claims, $s$ converges to an NE after at most three while loops.

Calculating the optimal social cost is also required to measure the efficiency of NEs. This problem is NP-hard, since it is equivalent to the minimum $k$-dominating set problem, which is known to be NP-hard [Hedetniemi and Laskar, 1991]. Fortunately, we can easily formulate the problem as an integer program, and solve it using intlinprog in MATLAB.

\subsection{Datasets}

We run simulations on the following networks:

- Synthetic:

- Star (100 nodes, 99 edges).

- Chain (100 nodes, 99 edges).

- Random (50 nodes, 127 edges): an Erdôs-Rényi random graph $G(50,0.1)$ (i.e., 50 nodes and each

- Real: edge exists i.i.d. with probability 0.1 ).

- Karate club (34 nodes, 78 edges): a friendship network between the members of a karate club at a university [Zachary, 1977].

- Hamsterster (1,858 nodes, 12,534 edges): a friendship network between the users of Hamsterster, an online community for hamster owners.

- Advogato (5,155 nodes, 51,127 edges): a trust network between the users of Advogato, an online community for programmers [Massa et al., 2009].

\subsection{Simulation Results}

In Table 4, social costs of social optima, NEs in SGGs, and NEs in SGG-ACs are compared. For NEs, we report the average social cost of 1,000 NEs returned by Algorithm 1 . The price $p$ of a good, which is simply a scale factor, is set to 1 .

Inefficiency of NEs in SGG. As seen in Table 4, the largest inefficiency (i.e., social cost in NEs / the optimal cost) 97.6 is obtained on the star graph. This is because the efficient NE, where only the center node buys a good, is unlikely to be realized, as the center node loses the incentive to buy a good as soon as any of the others does. Thus, the inefficient NE, where all nodes except the center node buy a good, is realized with high probability. On the chain graph and the random graph, however, the inefficiency is only 1.28 and 1.55 , respectively, since high-degree nodes, which are main source of inefficiency, do not exist. On real networks, where highdegree nodes exist (albeit not as extreme as the star graph), the inefficiency is between 3.28 and 4.25 .

Effects of access costs on reducing the social inefficiency. As seen in Table 4, the inefficiency significantly decreases in SGG-ACs with an appropriate access cost, compared to SGGs. In particular, on the star graph, the inefficiency decreases by $93 \%$. This is because the inefficient 
Proceedings of the Twenty-Sixth International Joint Conference on Artificial Intelligence (IJCAI-17)

Table 4: Social costs when $k=1$. The numbers in the parentheses indicate the standard deviations.

\begin{tabular}{c|c|c|c|c|c|c|c}
\hline \multirow{2}{*}{ Dataset } & \multicolumn{7}{|c}{ Social Cost } \\
\cline { 2 - 8 } & \multirow{2}{*}{ Optimum Cost } & \multirow{2}{*}{ NEs in SGGs } & \multicolumn{5}{|c}{ NEs in SGG-ACs } \\
\cline { 4 - 8 } & & $\xi=1$ & $\xi=2$ & $\xi=5$ & $\xi=10$ & $\xi=20$ \\
\hline Star & 1 & $97.6(9.50)$ & $\mathbf{1 . 6 9 ( 1 0 . 9 )}$ & $3.06(12.6)$ & $5.92(21.4)$ & $10.2(28.6)$ & $20.5(39.1)$ \\
Chain & 34 & $43.5(1.37)$ & $45.5(1.65)$ & $\mathbf{4 3 . 5 ( 1 . 3 6 )}$ & $43.5(1.38)$ & $43.5(1.35)$ & $43.5(1.38)$ \\
Random & 11 & $17.0(1.92)$ & $18.9(1.46)$ & $\mathbf{1 5 . 9 ( 1 . 7 0 )}$ & $17.1(1.91)$ & $17.1(1.90)$ & $17.1(1.90)$ \\
\hline Karate club & 4 & $17.0(3.31)$ & $10.2(1.73)$ & $\mathbf{8 . 4 6 ( 3 . 1 4 )}$ & $14.3(4.80)$ & $17.0(3.36)$ & $17.1(3.26)$ \\
Hamsterster & 241 & $970(23.6)$ & $526(12.4)$ & $\mathbf{4 2 6 ( 1 6 . 3 )}$ & $581(36.8)$ & $827(49.8)$ & $956(32.1)$ \\
Advogato & 806 & $2643(27.1)$ & $1468(22.5)$ & $\mathbf{1 1 9 6 ( 3 0 . 7 )}$ & $1475(56.2)$ & $1887(82.6)$ & $2199(101)$ \\
\hline
\end{tabular}

Table 5: Social costs when $k>1$.

\begin{tabular}{c|c|c|c|c}
\hline \multirow{2}{*}{ Dataset } & \multirow{2}{*}{ Outcomes } & \multicolumn{3}{|c}{ Social Cost } \\
\cline { 3 - 5 } & & $k=2$ & $k=3$ & $k=4$ \\
\hline \multirow{3}{*}{ Karate club } & Optimum Cost & 2 & 1 & 1 \\
& NEs in SGGs & 3.23 & 1.76 & 1.28 \\
& NEs in SGG-ACs & 3.23 & 1.75 & 1.26 \\
\hline \multirow{3}{*}{ Hamsterster } & Optimum Cost & 65 & 32 & 28 \\
& NEs in SGGs & 188 & 90.7 & 46.3 \\
& NEs in SGG-ACs & 128 & 65.0 & 41.7 \\
\hline \multirow{3}{*}{ Advogato } & Optimum Cost & 156 & 69 & 58 \\
& NEs in SGGs & 670 & 283 & 102 \\
& NEs in SGG-ACs & 377 & 129 & 86.5 \\
\hline
\end{tabular}

NE, where all nodes except the center node buy the good, is unlikely to be realized, as the center node can still buy a good even when it has neighbors buying goods. For similar reasons, the inefficiency on real networks also decreases by $50 \%-56 \%$. Since the inefficiency on the chain and random graphs is already low in SGGs, the improvement is smaller.

Access costs that maximize efficiency. As seen in Table 4, inefficiency is lowest at $\xi=2(p / 3<a<p / 2)$, consistent with our suggestion of $\xi=\max (2\lfloor k / 2\rfloor+1,2)$ in Section 3 . The inefficiency tends to increase as $\xi$ increases.

Effects of $k$. Social costs on real networks when $k>1$ are shown in Table 5, where we set $\xi$ to 6 on Karate club and 4 on the others. In both SGGs and SGG-ACs, inefficiency in NEs decreases as $k$ increases, which is consistent with Theorems 2, 3, and 4. Although the inefficiency is still smaller in SGG-ACs than in SGGs, the gap decreases as $k$ increases.

\section{Related Work}

We first review previous work directly related to network games with shareable goods. See the work of Galeotti et al. [2010], and the chapter by Jackson and Zenou [2014], for an introduction to network games in general.

Bramoullé and Kranton [2007] and Bramoullé et al. [2014] study a network game where the strategy of each node is its contribution to a public good, and its utility is a function of its own contribution and that of its direct neighbors.

Ballester et al. [2006] consider a similar game where, however, the utility of each node is a concave function of its effort and a linear function of its neighbors'. With these conditions, the game has a unique NE where the effort of each node is proportional to its Bonacich centrality score. Elliott and Golub [2013] study an extended game in directed, weighted graphs where an edge $(i, j)$ indicates the marginal benefit that node $i$ can provide to node $j$. Allouch [2015] develops a model where consumptions of both private goods and public goods are taken into account.

In our models (of Section 2), each node simply decides whether to buy a good or access its neighbors', rather than the amount of effort. Instead, we extend the previous models, especially the best-shot game [Hirshleifer, 1983], in that (a) access costs can be imposed on free riders and (b) nodes can benefit not only from direct neighbors but also $k$-hop neighbors. In contrast to previous work, we analyze PoA and PoS, and provide empirical results on real social networks.

Our work is also related to the huge body of literature on the price of anarchy. The concept itself is due to Koutsoupias and Papadimitriou [1999], and the price of stability was introduced a few years later by Anshelevich et al. [2004]. These concepts underlie much work at the intersection of game theory and AI, e.g., in computational social choice [Brânzei et al., 2013], security games [Lou and Vorobeychik, 2015], and routing [Vasserman et al., 2015].

To the best of our knowledge, the price-of-anarchy paper that is most closely related to ours is the one by Kun et al. [2013]. They give bounds on the price of anarchy of an anti-coordination game played on a graph, albeit a fundamentally different one: each player chooses a color, and the utility of a player is the number of neighbors with different colors. In their work, $k$-hop neighbors are not considered, and access costs are incompatible with the model. It is also worth mentioning that our use of access costs to reduce the inefficiency of equilibria is conceptually related to work on taxation in congestion games [Caragiannis et al., 2010].

\section{Final Words}

In our view, the most actionable conclusion from our work is that in the type of scenarios under consideration (shareable goods on a network), access costs should be imposed when possible. Despite the whimsical title of our paper, this would be hard to do at a societal level for things like ski equipment and portable cribs. However, it certainly seems feasible at the level of an organization. For example, a university could mandate access costs for expensive lab equipment bought by individual researchers, as this would actually decrease the amount of grant money that is invested in buying equipment. For the designer of a multi-agent system, imposing access costs is trivial, and, similarly, might lead (would lead, if one trusts our analysis) to significant benefits.

\section{Acknowledgements}

We thank Prof. Christos Faloutsos for fruitful discussions. 


\section{References}

[Allouch, 2015] N. Allouch. On the private provision of public goods on networks. Journal of Economic Theory, 157:527-552, 2015.

[Anshelevich et al., 2004] E. Anshelevich, A. Dasgupta, J. M. Kleinberg, É. Tardos, T. Wexler, and T. Roughgarden. The price of stability for network design with fair cost allocation. In Proceedings of the 45th Symposium on Foundations of Computer Science (FOCS), pages 295304, 2004.

[Ballester et al., 2006] C. Ballester, A. Calvó-Armengol, and Y. Zenou. Who's who in networks. Wanted: the key player. Econometrica, 74(5):1403-1417, 2006.

[Blume, 1993] L. E. Blume. The statistical mechanics of strategic interaction. Games and economic behavior, 5(3):387-424, 1993.

[Bramoullé and Kranton, 2007] Y. Bramoullé and R. Kranton. Public goods in networks. Journal of Economic Theory, 135(1):478-494, 2007.

[Bramoullé et al., 2014] Y. Bramoullé, R. Kranton, and M. D'amours. Strategic interaction and networks. The American Economic Review, 104(3):898-930, 2014.

[Brânzei et al., 2013] S. Brânzei, I. Caragiannis, J. Morgenstern, and A. D. Procaccia. How bad is selfish voting? In Proceedings of the 27th AAAI Conference on Artificial Intelligence (AAAI), pages 138-144, 2013.

[Caragiannis et al., 2010] I. Caragiannis, C. Kaklamanis, and P. Kanellopoulos. Taxes for linear atomic congestion games. ACM Transactions on Algorithms, 7(1): article 13, 2010.

[Elliott and Golub, 2013] M. Elliott and B. Golub. A network approach to public goods. In Proceedings of the 14th ACM Conference on Economics and Computation (EC), pages 377-378, 2013.

[Ellison, 1993] G. Ellison. Learning, local interaction, and coordination. Econometrica, 61(5):1047-1071, 1993.

[Galeotti et al., 2010] A. Galeotti, S. Goyal, M. O. Jackson, F. Vega-Redondo, and L. Yariv. Network games. The review of economic studies, 77(1):218-244, 2010.

[Hedetniemi and Laskar, 1991] S. T. Hedetniemi and R. C. Laskar. Topics on Domination. Elsevier, 1991.

[Hirshleifer, 1983] J. Hirshleifer. From weakest-link to bestshot: The voluntary provision of public goods. Public choice, 41(3):371-386, 1983.
[Jackson and Zenou, 2014] M. O Jackson and Y. Zenou. Games on networks. In Handbook of Game Theory, volume 4. North-Holland, 2014.

[Koutsoupias and Papadimitriou, 1999] E. Koutsoupias and C. H. Papadimitriou. Worst-case equilibria. In Proceedings of the 16th International Symposium on Theoretical Aspects of Computer Science (STACS), pages 404-413, 1999.

[Kreuter and Nierhoff, 1997] B. Kreuter and T. Nierhoff. Greedily approximating the $r$-independent set and $k$ center problems on random instances. In Proceedings of the International Workshop on Randomization and Approximation Techniques in Computer Science (RANDOM), pages 43-53, 1997.

[Kun et al., 2013] J. Kun, B. Powers, and L. Reyzin. Anticoordination games and stable graph colorings. In Proceedings of the 6th International Symposium on Algorithmic Game Theory (SAGT), pages 122-133, 2013.

[Lou and Vorobeychik, 2015] J. Lou and Y. Vorobeychik. Equilibium analysis of multi-defender security games. In Proceedings of the 24th International Joint Conference on Artificial Intelligence (IJCAI), pages 596-602, 2015.

[Markus, 1987] M. L. Markus. Toward a "critical mass" theory of interactive media universal access, interdependence and diffusion. Communication research, 14(5):491-511, 1987.

[Massa et al., 2009] P. Massa, M. Salvetti, and D. Tomasoni. Bowling alone and trust decline in social network sites. In Proceedings of the 8th IEEE International Conference on Dependable, Autonomic and Secure Computing (DASC), 2009.

[Matsui, 1992] A. Matsui. Best response dynamics and socially stable strategies. Journal of Economic Theory, 57(2):343-362, 1992.

[Rogers, 2010] E. M. Rogers. Diffusion of Innovations. Simon and Schuster, 2010.

[Shin et al., 2017] K. Shin, E. Lee, D. Eswaran, and A. D Procaccia. Why you should charge your friends for borrowing your stuff. arXiv preprint arXiv:1705.07343, 2017.

[Vasserman et al., 2015] S. Vasserman, M. Feldman, and A. Hassidim. Implementing the wisdom of Waze. In Proceedings of the 24th International Joint Conference on Artificial Intelligence (IJCAI), pages 660-666, 2015.

[Zachary, 1977] W. W. Zachary. An information flow model for conflict and fission in small groups. Journal of Anthropological Research, 33(4):452-473, 1977. 